\title{
IL-6 polymorphisms: a useful genetic tool for inflammation research?
}

\author{
Patricia Woo' and Steve E. Humphries ${ }^{2}$ \\ ${ }^{1}$ Centre for Paediatric and Adolescent Rheumatology, Division of Infection and Immunity, and ${ }^{2}$ Centre for Cardiovascular Genetics, \\ UCL Institute of Cardiovascular Science, University College London, London, United Kingdom.
}

In 1998, we described a novel polymorphism in the promoter $(G>C$, rs1800795) of the IL-6 (IL6) gene. The common allele, G, exhibited higher transcriptional activity in gene reporter assays and was associated with higher serum IL-6 levels in a small cohort of healthy subjects. We explored the ethnic distribution of these alleles and found significant differences among people of mixed European descent, Africans, and Gujarati Asians. Disease association was established in a cohort of 92 children of mixed European descent from the United Kingdom with systemic juvenile idiopathic arthritis (sJIA), with the GG genotype being significantly increased in sJIA cases compared with that in 383 controls, especially in those under 6 years old $(P=0.01)$. This polymorphism has since been used as a functional variant to explore the role of elevated IL-6 levels in many common disease states, confirming the key causal role of IL-6 in human health and disease.

\section{Introduction}

Collaborations are most fruitful when both parties bring complementary expertise but have a common/shared interest. For both of us, working in different buildings in University College London (UCL), the common interest was in identifying DNA variants that influence expression of key inflammatory cytokines, while the complementary interests were in cell and molecular biology of cytokines in systemic juvenile idiopathic arthritis (sJIA) and in molecular genetics and genetic epidemiology of coronary artery disease (CAD). At that time it was already clear that overexpression of IL- 6 was likely to be central to the pathogenesis of the chronic systemic inflammation seen in young children with sJIA. Similarly, there had been growing recognition of the key role of inflammation in the development of atherosclerosis, with the landmark paper from the late Russell Ross in 1999 (1).

The obvious place to look for functional variants affecting expression of IL6 was in the proximal promoter of the gene. These studies were carried out in a small cohort of normal healthy individuals by Daniel Fishman, a clinician studying for his $\mathrm{PhD}$ in the Woo laboratory (2). His

Conflict of interest: The authors have declared that no conflict of interest exists.

Citation for this article: J Clin Invest. 2013; 123(4):1413-1414. doi:10.1172/JCI67221. findings set off a series of experiments to assess whether the identified variants affected gene expression, and he found that the variant in position $-174 \mathrm{bp}$ (subsequently designated as rs1800795) upstream of the transcriptional start site did in fact affect IL6 gene transcription. Furthermore, the common genotype in people of mixed European descent, GG, was significantly associated with sJIA cases when compared with the pool of controls from the Humphries laboratory. Subsequent analysis of the 5'-flanking region of IL 6 by our groups at UCL identified further genetic variants up to $6 \mathrm{~kb}$ from the start of transcription that influence expression and are associated with serum levels (3) and constitutive expression (4). Most recently, we reported on a chromatin loop structure involving this $-6-\mathrm{kb}$ region that interacts directly with the proximal promoter region through OCT1 binding (5). We are still working to identify the additional nuclear proteins involved in the exquisite control of this intriguing cytokine.

\section{Genetic variants: a useful tool to probe the cause of inflammation}

The discovery of a functional polymorphism in the IL6 gene led to a large number of candidate gene association studies in different inflammatory diseases. The problem with the SNP genetic association studies reported in the 1990 s was that all of them were underpowered to detect the modest effects on a trait that would be expected for a common variant. As a consequence, all were tarred with the "false-positive" brush (6). Indeed, some subsequent papers did not confirm the association with IL-6 levels in healthy subjects, but many did see this in patients undergoing an inflammatory stimulus such as after coronary artery bypass surgery (7) or an operation for periodontal gum disease (3). Diseases associated with raised IL-6 levels are numerous, including CAD and abdominal aortic aneurysms (AAA) as well as autoimmune diseases, such as systemic lupus erythematosis and rheumatoid arthritis. The number of inflammatory diseases with evidence of higher than normal IL-6 levels in serum or in tissues is growing (see Supplemental Table 1; supplemental material available online with this article; doi:10.1172/JCI67221DS1). The accurate measurement of IL-6 in serum is complicated by the fact that IL- 6 levels vary in a diurnal manner in the healthy individual, so the time of sampling would affect the final results. Unfortunately, none of the papers describing serum IL-6 levels provide data on time of collection or time to storage and length of storage.

In many cases, the increase in IL-6 levels is believed to be secondary to other inflammatory mediators such as IL-1 and TNF. The key question is whether there is a genetic predisposition in the disease under investigation to produce higher IL-6 levels, leading to a chronic proinflammatory state. In retrospect, it was remarkable that we observed a significant association of $-174 \mathrm{G}>\mathrm{C}$ with the serum IL-6 level in the healthy subjects, and the "winner's curse" may explain the smaller effects reported subsequently. However, the identification of a functional variant, which because of disequilibrium marks a functional haplotype of IL6 promoter variants, has allowed this SNP to be used as a genetic instrument to probe the causality of higher IL- 6 levels in many different disease states, an 
A

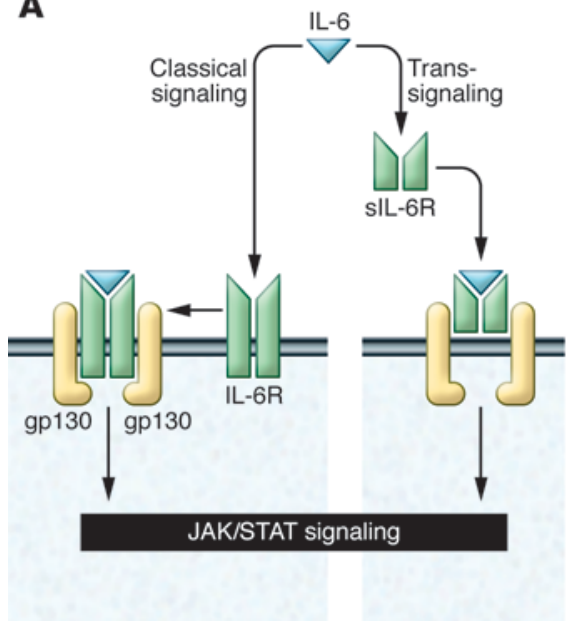

approach known as Mendelian randomization (8). Further, this variant has been used to probe the influence of elevated IL-6 levels in many disease-state meta-analyses, some of which include more than 100,000 subjects (see Supplemental Table 1 ), ensuring the statistical robustness of the associations.

\section{Clinical relevance of IL6 polymorphisms}

Confirmation of disease association of the IL6 gene with sJIA was obtained in a study of families from the United Kingdom and America that used the transmission disequilibrium test (9). Since then, multiple genes have been associated with sJIA, likely reflecting multiple pathogenetic factors leading to the same clinical syndrome (reviewed in ref. 10). Currently, an international consortium is carrying out a whole-genome scan, but even with worldwide collection of genotypes from people of mixed European origins, the numbers still fall short of that required for a conventional, statistically acceptable result. It is possible that with the availability of exome sequencing and next-generation whole-genome sequencing the genetic architecture of sJIA may be unravelled within the next few years.

The discovery of the association of IL6 with sJIA lent considerable weight to the hypothesis that IL- 6 is central to the pathology of this disease and led to the clinical trial of an IL- 6 receptor (IL-6R) monoclonal antibody (see Figure 1 ) to block IL-6 classical and trans-signaling in an orphan disease such as sJIA. Despite the "weak" genetic association, this antibody (tocilizumab) has proven to have

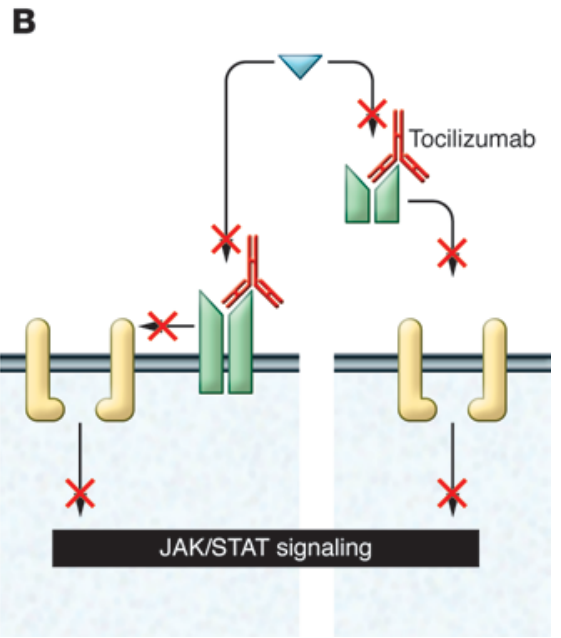

\section{Figure 1}

Mechanism of action of tocilizumab. (A) IL-6 triggers the dimerization of gp130 through binding of IL-6R. (B) Tocilizumab binds to the IL-6R or the soluble IL-6R (sIL-6R), which mediates IL- 6 trans-signaling, preventing the binding of IL- 6 . Loss of IL- 6 binding reduces both classical and trans-signaling and reduces activation of the JAK/STAT system, leading to damping of inflammation locally and systemically. remarkable efficacy in this disease in a proof-of-principle phase I/II study with a single dose and in a separate dose-escalation study $(11,12)$. The subsequent multinational phase III double-blind placebo-controlled trial achieved the primary outcome in $85 \%$ of treated cases compared with $24 \%$ in the placebo group at week 12 , and $80 \%$ of treated patients showed $70 \%$ improvement at week 52 (13). In rheumatoid arthritis, the antibody's efficacy is around $60 \%$ to $65 \%$. Tocilizumab is now approved as a second-line treatment agent for sJIA by the FDA and European Medicines Agency. The finding that common IL6R SNPs that are associated with low levels of systemic inflammation are also strongly predictive of CAD (14) and AAA (15) suggests that agents such as tocilizumab may be beneficial for treatment of these diseases as well.

\section{Acknowledgments}

We thank all members of our research team over the last 15 years for their hard work and creative contributions to the IL-6 story. P. Woo is supported by the MRC and ARUK. S.E. Humphries is supported by the British Heart Foundation (PG08/008).

Address correspondence to: Patricia Woo, 3rd floor, Rayne Building, University College London, 5, University Street, London, WC1E 6JF, United Kingdom. Phone: 0207.679.6364; Fax: 0207.679.6212; E-mail: patricia.woo@ucl.ac.uk.

1. Ross R. Atherosclerosis - an inflammatory disease. NEngl J Med. 1999;340(2):115-126.

2. Fishman D, et al. The effect of novel polymorphisms in the interleukin-6 (IL-6) gene on IL-6 transcription and plasma IL-6 levels, and an asso- ciation with systemic-onset juvenile chronic arthritis. J Clin Invest. 1998;102(7):1369-1376.

3. Smith AJ, et al. Association of serum interleukin- 6 concentration with a functional IL6 -6331T >C polymorphism. Clin Chem. 2008;54(5):841-850.

4. Samuel JM, Kelberman D, Smith AJ, Humphries SE, Woo P. Identification of a novel regulatory region in the interleukin- 6 gene promoter. Cytokine. 2008;42(2):256-264.

5. Smith AJ, Zheng D, Palmen J, Pang DX, Woo P, Humphries SE. Effects of genetic variation on chromatin structure and the transcriptional machinery: analysis of the IL6 gene locus. Genes Immun. 2012;13(7):583-586.

6. Ioannidis JP. Why most published research findings are false. PLoS Med. 2005;2(8):e124.

7. Brull DJ, et al. Interleukin-6 gene $-174 \mathrm{~g}>\mathrm{c}$ and $-572 \mathrm{~g}>\mathrm{c}$ promoter polymorphisms are strong predictors of plasma interleukin-6 levels after coronary artery bypass surgery. Arterioscler Thromb Vasc Biol. 2001;21(9):1458-1463.

8. Hingorani A, Humphries S. Nature's randomised trials. Lancet. 2005;366(9501):1906-1908.

9. Ogilvie EM, et al. The $-174 \mathrm{G}$ allele of the interleukin- 6 gene confers susceptibility to systemic arthritis in children: a multicenter study using simplex and multiplex juvenile idiopathic arthritis families. Arthritis Rheum. 2003;48(11):3202-3206.

10. Prahalad S, Glass DN. A comprehensive review of the genetics of juvenile idiopathic arthritis. Pediatr Rheumatol Online J. 2008;6:11.

11. Yokota $\mathrm{S}$, et al. Therapeutic efficacy of humanized recombinant anti-interleukin- 6 receptor antibody in children with systemic-onset juvenile idiopathic arthritis. Arthritis Rheum. 2005; 52(3):818-825

12. Woo P, et al. Open label phase II trial of single, ascending doses of MRA in Caucasian children with severe systemic juvenile idiopathic arthritis: proof of principle of the efficacy of IL- 6 receptor blockade in this type of arthritis and demonstration of prolonged clinical improvement. Arthritis Res Ther. 2005;7(6):R1281-R1288.

13. De Benedetti F, et al. Randomized trial of tocilizumab in systemic juvenile idiopathic arthritis. NEngl J Med. 2012;367(25):2385-2395.

14. Hingorani AD, Casas JP. The interleukin-6 receptor as a target for prevention of coronary heart disease: a mendelian randomisation analysis. Lancet. 2012;379(9822):1214-1224.

15 . Harrison SC, et al. Interleukin- 6 receptor pathways in abdominal aortic aneurysm [published online of print October 30, 2012]. Eur Heart J. doi:10.1093/ eurheartj/ehs354. 